\title{
DIFFERENCE EQUATIONS ON DISCRETE POLYNOMIAL HYPERGROUPS
}

ÁGOTA OROSZ

Received 10 July 2005; Revised 24 October 2005; Accepted 30 October 2005

The classical theory of homogeneous and inhomogeneous linear difference equations with constant coefficients on the set of integers or nonnegative integers provides effective solution methods for a wide class of problems arising from different fields of applications. However, linear difference equations with nonconstant coefficients present another important class of difference equations with much less highly developed methods and theories. In this work we present a new approach to this theory via polynomial hypergroups. It turns out that a major part of the classical theory can be converted into hypergroup language and technique, providing effective solution methods for a wide class of linear difference equations with nonconstant coefficients.

Copyright (C 2006 Ágota Orosz. This is an open access article distributed under the Creative Commons Attribution License, which permits unrestricted use, distribution, and reproduction in any medium, provided the original work is properly cited.

\section{Introduction}

A linear difference equation with nonconstant coefficients has the following general form:

$$
a_{N}(n) f_{n+N}+a_{N-1}(n) f_{n+N-1}+\cdots+a_{1}(n) f_{n+1}+a_{0}(n) f_{n}=g_{n}
$$

where the functions $a_{0}, a_{1}, \ldots, a_{N}, g: \mathbb{N} \rightarrow \mathbb{C}$ are given with $a_{N}$ not identically zero, and $N, k$ are fixed nonnegative integers (in this paper $\mathbb{N}=\{0,1,2, \ldots\}$ ). The above equation is supposed to hold for some unknown function $f: \mathbb{N} \rightarrow \mathbb{C}$ or $f: \mathbb{Z} \rightarrow \mathbb{C}$, depending on the nature of the problem. In what follows we will prefer the case $f: \mathbb{N} \rightarrow \mathbb{C}$ and the notation $f(m)$ and $g(m)$ instead of $f_{m}$ and $g_{m}$.

By the classical theory of differential equations the solution space of the above equation can be described completely in the constant coefficient case, that is, if the functions $a_{0}, a_{1}, \ldots, a_{N}$ are constants. In this case the solution space is generated by exponential monomial solutions, which arise from the roots of the characteristic polynomial, called characteristic roots. 
Much less is known in the case of nonconstant coefficients. In this work we offer a method to solve some types of homogeneous linear difference equations with nonconstant coefficients by transforming these equations into homogeneous linear difference equations with constant coefficients over hypergroups. This method is based on some theory of homogeneous linear difference equations with constant coefficients on hypergroups developed along the lines of the classical theory over $\mathbb{N}$. The basic idea is that the role of exponential functions is played by the generating polynomials of some polynomial hypergroups.

Some results of this work have been presented at the 5th Debrecen-Katowice Winter Seminar in Będlewo (Poland) in 2005.

We remark that most of this work can be generalized to the case of signed hypergroups, as they are presented in [1]. Nevertheless, in the forthcoming presentation we restrict ourselves to polynomial hypergroups in the sense of [2].

\section{Discrete polynomial hypergroups}

Let $\left(\alpha_{n}\right)_{n \in \mathbb{N}},\left(\beta_{n}\right)_{n \in \mathbb{N}}$ and $\left(\gamma_{n}\right)_{n \in \mathbb{N}}$ be real sequences with the following properties: $\gamma_{n}>0$, $\beta_{n} \geq 0, \alpha_{n+1}>0$ for all $n$ in $\mathbb{N}$, moreover $\alpha_{0}=0$, and $\alpha_{n}+\beta_{n}+\gamma_{n}=1$ for all $n$ in $\mathbb{N}$. We define the sequence of polynomials $\left(P_{n}\right)_{n \in \mathbb{N}}$ by $P_{0}(x)=1, P_{1}(x)=x$, and by the recursive formula

$$
x P_{n}(x)=\alpha_{n} P_{n-1}(x)+\beta_{n} P_{n}(x)+\gamma_{n} P_{n+1}(x)
$$

for all $n \geq 1$ and $x$ in $\mathbb{R}$. In this case there exists constants $c(n, m, k)$ for all $n, m, k$ in $\mathbb{N}$ such that

$$
P_{n} P_{m}=\sum_{k=|n-m|}^{n+m} c(n, m, k) P_{k}
$$

holds for all $n, m$ in $\mathbb{N}$ (see $[3,4]$ ). This formula is called linearization formula, and the coefficients $c(n, m, k)$ are called linearization coefficients. It is clear that $P_{n}(1)=1$ for all $n$ in $\mathbb{N}$, hence we have

$$
\sum_{k=|n-m|}^{n+m} c(n, m, k)=1
$$

for all $n$ in $\mathbb{N}$. If the linearization coefficients are nonnegative: $c(n, m, k) \geq 0$ for all $n, m, k$ in $\mathbb{N}$, then we can define a hypergroup structure on $\mathbb{N}$ by identifying the natural numbers with the Dirac-measures in virtue of the following rule:

$$
\delta_{n} * \delta_{m}=\sum_{k=|n-m|}^{n+m} c(n, m, k) \delta_{k}
$$

for all $n, m$ in $\mathbb{N}$, with involution as the identity mapping and with $e$ as 0 . The resulting hypergroup $K=(\mathbb{N}, *)$ is called the polynomial hypergroup associated with the sequence $\left(P_{n}\right)_{n \in \mathbb{N}}$. 
Let $f: \mathbb{N} \rightarrow \mathbb{C}$ is an arbitrary function and $m$ is a natural number. The translate of $f$ by $m$ is defined by

$$
\mathscr{T}_{m} f(n)=\int_{\mathbb{N}} f(k) d\left(\delta_{n} * \delta_{m}\right)(k)
$$

for all $n$ in $\mathbb{N}$. Although $n * m$ is not defined on the hypergroup, the following notation is in use:

$$
f(n * m)=\mathscr{T}_{m} f(n)=\sum_{k=|n-m|}^{n+m} c(n, m, k) f(k)
$$

for each $n, m$ in $\mathbb{N}$. The function $\chi: \mathbb{N} \rightarrow \mathbb{C}$ is said to be an exponential function on the polynomial hypergroup if

$$
\chi(n * m)=\chi(n) \chi(m)
$$

holds for all $n, m$ in $\mathbb{N}$. If the hypergroup is generated by the sequence of polynomials $\left(P_{n}\right)_{n \in \mathbb{N}}$, then a function $\chi: \mathbb{N} \rightarrow \mathbb{C}$ is an exponential function if and only if

$$
\chi(n)=P_{n}(\lambda)
$$

holds for some complex number $\lambda$ (see [2]).

\section{Difference equations with 1-translation}

In the classical theory of difference equations the translate of a function by $n$ and the translation of the function $n$-times by 1 give the same result for all $n$ in $\mathbb{N}$. But in the hypergroup case there are two different ways to define difference equations along these two interpretations. In this section we deal with the latter one. We introduce the notation

$$
\mathscr{T} f(n)=\mathscr{T}_{1} f(n)=f(n * 1)
$$

for any $f: \mathbb{N} \rightarrow \mathbb{C}$ and $n$ in $\mathbb{N}$, moreover $\mathscr{T}^{0} f=f$ and $\mathscr{T}^{N} f=\mathscr{T}\left(\mathscr{T}^{N-1} f\right)$ for each integer $N>1$. Obviously, $\mathscr{T}$ is a linear operator on the linear space $\mathbb{C}^{\mathbb{N}}$ of all complex valued functions on $\mathbb{N}$. If $Q$ is any polynomial with complex coefficients, then $Q(\mathscr{T})$ has the obvious meaning. Let $N$ be a positive integer, $a_{0}, \ldots, a_{N}$ be complex numbers and suppose that $a_{N} \neq 0$. We will consider functional equations of the form

$$
Q(\mathscr{T}) f=a_{N} \mathscr{T}^{N} f(n)+a_{N-1} \mathscr{T}^{N-1} f(n)+\cdots+a_{0} f(n)=0,
$$

which is called a homogeneous linear difference equation of order $N$ on the hypergroup $K$ with constant coefficients associated to the polynomial $Q$. The polynomial $Q$ is called the characteristic polynomial of (3.2) and its roots are called the characteristic roots of (3.2). The solution space of (3.2) is the kernel of the linear operator $Q(\mathscr{T})$, hence it is a linear subspace of the function space $\mathbb{C}^{\mathbb{N}}$. This solution space is translation invariant in the sense that if $f$ is a solution, then $\mathscr{T} f$ is a solution, too. 
4 Difference equations on discrete polynomial hypergroups

THeORem 3.1. If $Q$ is a complex polynomial of degree $N \geq 1$, then the solution space of (3.2) has dimension $N$.

Proof. Suppose that $f: \mathbb{N} \rightarrow \mathbb{C}$ is a solution of (3.2). Since $f(0 * 1)=f(1)$ and for $n \geq 1$ we have

$$
f(n * 1)=\sum_{k=n-1}^{n+1} c(n, 1, k) f(k)=\alpha_{n} f(n-1)+\beta_{n} f(n)+\gamma_{n} f(n+1),
$$

where $\left(\alpha_{n}\right)_{n \in \mathbb{N}},\left(\beta_{n}\right)_{n \in \mathbb{N}}$ and $\left(\gamma_{n}\right)_{n \in \mathbb{N}}$ are the sequences appearing in the definition of the polynomial hypergroup. Considering (3.2) for $n=0$ we get

$$
a_{N} \gamma_{N-1} \cdots \gamma_{1} \gamma_{0} f(N)+\sum_{i=0}^{N-1} k_{N, i} f(i)=0
$$

with some complex numbers $k_{N, i}(i=0, \ldots, N-1)$. Since obviously $a_{N} \gamma_{N-1} \cdots \gamma_{0} \neq 0$, hence $f(N)$ is determined by $f(0), \ldots, f(N-1)$ and it is easy to see by induction that $f(n)$ is uniquely determined by the values $f(0), \ldots, f(N-1)$ for $n \geq N$.

THeOREм 3.2. If the complex number $\lambda$ is a characteristic root of (3.2) with multiplicity $m$, then all the functions $n \mapsto P_{n}^{(k)}(\lambda)$ are solutions of (3.2) for $k=0,1, \ldots, m-1$.

Proof. By the exponential property of the function $n \mapsto P_{n}(\lambda)$ we have

$$
\mathscr{T} P_{n}(\lambda)=P_{n}(\lambda) P_{1}(\lambda)=\lambda P_{n}(\lambda), \quad \mathscr{T}^{t} P_{n}(\lambda)=\lambda^{t} P_{n}(\lambda),
$$

thus we can see immediately that this function is a solution of (3.2):

$$
Q(\mathscr{T}) P_{n}(\lambda)=\left(\lambda^{N}+a_{N-1} \lambda^{N-1}+\cdots+a_{1} \lambda+a_{0}\right) P_{n}(\lambda)=0 .
$$

For proving that $n \mapsto P_{n}^{(k)}(\lambda)$ are also solutions for $1 \leq k \leq m$, we need the translates of $P_{n}^{(k)}(\lambda)$. After some calculation we get that

$$
\mathcal{T}^{r} P_{n}^{(k)}(\lambda)=\sum_{t=0}^{\min (r, k)}\left(\begin{array}{l}
r \\
t
\end{array}\right) \lambda^{r-t} \frac{k !}{(k-t) !} P_{n}^{(k-t)}(\lambda)
$$

for $r$ in $\mathbb{N}$, therefore we have

$$
\begin{aligned}
Q(\mathscr{T}) P_{n}^{(k)}(\lambda) & =\sum_{r=0}^{N} a_{r} \mathscr{T}^{r} P_{n}^{(k)}(\lambda) \\
& =\sum_{r=0}^{N} a_{r}\left(\sum_{t=0}^{\min (r, k)}\left(\begin{array}{l}
r \\
t
\end{array}\right) \lambda^{r-t} \frac{k !}{(k-t) !} P_{n}^{(k-t)}(\lambda)\right) \\
& =\sum_{t=0}^{k}\left(\begin{array}{l}
k \\
t
\end{array}\right)\left(\sum_{r=t}^{N} a_{r} \frac{r !}{(r-t) !} \lambda^{r-t}\right) P_{n}^{(k-t)}(\lambda)=0
\end{aligned}
$$

as the $t$ th derivative of the characteristic polynomial at $\lambda$ is equal to zero for $0 \leq t \leq$ $m-1$. 
Lemma 3.3. Let $k$ be a positive integer and $l_{1}, \ldots, l_{k}$ nonnegative integers. If $\lambda_{1}, \ldots, \lambda_{k}$ are different complex numbers, then the functions $P_{n}^{(i)}\left(\lambda_{j}\right)$ are linearly independent for $j=$ $1,2, \ldots, k$ and $i=0,1, \ldots, l_{j}$.

Proof. First we show that $P_{n}\left(\lambda_{1}\right), \ldots, P_{n}\left(\lambda_{k}\right)$ are linearly independent if $\lambda_{1}, \ldots, \lambda_{k}$ are different. If it is not the case, then there are complex numbers $a_{1}, a_{2}, \ldots, a_{k}$, not all equal to zero, with the property $\sum_{i=1}^{k} a_{i} P_{n}\left(\lambda_{i}\right)=0$, which contradicts the fact that for some constant $C$ the following equation holds:

$$
\left|\begin{array}{ccc}
P_{0}\left(\lambda_{1}\right) & \cdots & P_{0}\left(\lambda_{k}\right) \\
P_{1}\left(\lambda_{1}\right) & \cdots & P_{1}\left(\lambda_{k}\right) \\
& \ddots & \\
P_{k}\left(\lambda_{1}\right) & \cdots & P_{k}\left(\lambda_{k}\right)
\end{array}\right|=C\left|\begin{array}{ccc}
1 & \cdots & 1 \\
\lambda_{1} & \cdots & \lambda_{k} \\
& \ddots & \\
\lambda_{1}^{k} & \cdots & \lambda_{k}^{k}
\end{array}\right| \neq 0 .
$$

Now assume that there exist $\lambda_{1}, \ldots, \lambda_{k}$ different complex numbers such that the functions $n \mapsto P_{n}^{(i)}\left(\lambda_{j}\right)$ are linearly dependent for $j=1,2, \ldots, k$ and $i=0,1, \ldots, l_{j}$ for some positive integers $l_{1}, \ldots, l_{k}$. Suppose that $k$ is the minimal positive integer with this property, and also suppose that $l_{1}+\cdots+l_{k}$ is minimal. It means, that there exist complex numbers $a_{j, i}$, not all equal to zero for $j=1, \ldots, k$ and $i=0, \ldots, l_{j}$ such that

$$
\sum_{j=1}^{k} \sum_{i=0}^{l_{j}} a_{j, i} P_{n}^{(i)}\left(\lambda_{j}\right)=0
$$

holds with $a_{j, l_{j}} \neq 0$. Translating (3.10) by 1 we have

$$
\sum_{j=1}^{k} \sum_{i=1}^{l_{j}} a_{j, i}\left(\lambda_{j} P_{n}^{(i)}\left(\lambda_{j}\right)+P_{n}^{(i-1)}\left(\lambda_{j}\right)\right)+\sum_{j=1}^{k} a_{j, 0} \lambda_{j} P_{n}\left(\lambda_{j}\right)=0
$$

and if we subtract (3.10) times $\lambda_{1}$ from this equation we get an expression which does not contain $P_{n}^{\left(l_{1}\right)}\left(\lambda_{1}\right)$ :

$$
\sum_{i=0}^{l_{1}-1} c_{1, i} P_{n}^{(i)}\left(\lambda_{1}\right)+\sum_{j=2}^{k} a_{j, l_{j}}\left(\lambda_{1}-\lambda_{j}\right) P_{n}^{\left(l_{j}\right)}\left(\lambda_{j}\right)+\sum_{j=2}^{k} \sum_{i=0}^{l_{j}-1} c_{j, i} P_{n}^{(i)}\left(\lambda_{j}\right)=0
$$

with some constants $c_{j, i}$, and this means that either $k$ or $l_{1}+\cdots+l_{k}$ was not minimal.

Using Theorems 3.1, 3.2 and Lemma 3.3 we can characterize the solution space of (3.2) completely.

Theorem 3.4. Let $Q$ be a complex polynomial of degree $N \geq 1$ with all different complex zeros $\lambda_{1}, \lambda_{2}, \ldots, \lambda_{k}$, where the multiplicity of $\lambda_{j}$ is $l_{j}(j=1,2, \ldots, k)$. Then the function $f$ : $\mathbb{N} \rightarrow \mathbb{C}$ is a solution of (3.2) if and only if it is a linear combination of functions of the form $n \mapsto P_{n}^{(i)}\left(\lambda_{j}\right)$ with $j=1,2, \ldots, k$ and $i=0,1, \ldots, l_{j}-1$. 
6 Difference equations on discrete polynomial hypergroups

\section{Difference equations with general translation}

Let us consider the following equation for a function $f: \mathbb{N} \rightarrow \mathbb{C}$

$$
a_{N} \mathscr{T}_{N} f(n)+a_{N-1} \mathscr{T}_{N-1} f(n)+\cdots+a_{0} f(n)=0
$$

where $N$ is a positive integer and $a_{N}, \ldots, a_{0}$ are complex numbers. We note, that (4.1) can be written in the form

$$
a_{N} f(n * N)+a_{N-1} f(n *(N-1))+\cdots+a_{0} f(n)=0 .
$$

It is easy to see, that the solution space of (4.1) is a linear subspace of $\mathbb{C}^{\mathbb{N}}$ with dimension $N$. We will show, that this solution space is generated by similar functions like in the case of (3.2), but the characteristic polynomial is different: it depends on the basic generating polynomials of the hypergroup.

Theorem 4.1. The function $f: \mathbb{N} \rightarrow \mathbb{C}$ is a solution of (4.1) if and only if it is the linear combination of functions of the form $n \mapsto P_{n}^{(i)}\left(\lambda_{j}\right)$ with $j=1,2, \ldots, k$ and $i=0,1, \ldots, l_{j}-1$, where $\lambda_{1}, \lambda_{2}, \ldots, \lambda_{k}$ are different complex zeros of the polynomial

$$
\lambda \longmapsto a_{N} P_{N}(\lambda)+a_{N-1} P_{N-1}(\lambda)+\cdots+a_{1} P_{1}(\lambda)+a_{0},
$$

and the multiplicity of $\lambda_{j}$ is $l_{j}(j=1,2, \ldots, k)$.

Proof. It will be sufficient to show, that the functions $n \mapsto P_{n}^{(i)}\left(\lambda_{j}\right)$ with $j=1,2, \ldots, k$ and $i=0,1, \ldots, l_{j}-1$ are solutions. Since

$$
\mathscr{T}_{m}\left(P_{n}^{(i)}(\lambda)\right)=\sum_{t=0}^{i}\left(\begin{array}{l}
i \\
t
\end{array}\right) P_{m}^{(t)}(\lambda) P_{n}^{(i-t)}(\lambda)
$$

for all $m$ in $\mathbb{N}$, substituting $P_{n}^{(i)}(\lambda)$ instead of $f(n)$ in (4.1) we get

$$
\begin{aligned}
\sum_{m=0}^{N} a_{m} \mathscr{T}_{m}\left(P_{n}^{(i)}(\lambda)\right) & =\sum_{m=0}^{N} a_{m} \sum_{t=0}^{i}\left(\begin{array}{l}
i \\
t
\end{array}\right) P_{m}^{(t)}(\lambda) P_{n}^{(i-t)}(\lambda) \\
& =\sum_{t=0}^{i}\left(\begin{array}{l}
i \\
t
\end{array}\right) P_{n}^{(i-t)}(\lambda)\left(\sum_{m=0}^{N} a_{m} P_{m}^{(t)}(\lambda)\right)=0,
\end{aligned}
$$

which holds if $\lambda$ is a root of (4.3) with a multiplicity higher than $i$.

\section{Examples}

Example 5.1. We consider the equation

$$
\mathscr{T} f=0 .
$$

On the Chebyshev-hypergroup we have

$$
\mathscr{T} f(n)=\frac{1}{2}(f(n+1)+f(|n-1|)),
$$


hence (5.1) has the form

$$
f(n+1)+f(|n-1|)=0
$$

for $n=0,1, \ldots$ With $n=0$ we have $f(1)=0$, and with $n \geq 0$ it follows $f(n+2)+f(n)=$ 0 , which implies $f(2 n+1)=0$ and $f(2 n)=(-1)^{n} f(0)$. On the Legendre-hypergroup we have

$$
\mathscr{T} f(n)=\frac{n+1}{2 n+1} f(n+1)+\frac{n}{2 n+1} f(|n-1|),
$$

hence (5.1) has the form

$$
(n+1) f(n+1)+n f(|n-1|)=0
$$

for $n \geq 0$. With $n=0$ we have $f(1)=0$, and with $n \geq 0$ it follows $(n+2) f(n+2)+(n+$ 1) $f(n)=0$, which implies $f(2 n+1)=0$, moreover $f(2 n)=(-1)^{n}((2 n-1) ! ! /(2 n) ! !) f(0)$. (Here $n$ !! denotes the double factorial of $n$.)

One observes that in the first case $f(n)=f(0) \cdot T_{n}(0)$ and in the second case $f(n)=$ $f(0) \cdot P_{n}(0)$, where $T_{n}$, respectively $P_{n}$ denotes the $n$th Chebyshev-polynomial, respectively the $n$th Legendre-polynomial. This is a simple consequence of our previous results. Indeed, the characteristic polynomial of $(5.1)$ is $Q(\lambda)=\lambda$, hence the only characteristic root is $\lambda=0$ with multiplicity 1 . Hence, on any polynomial hypergroup with generating polynomials $\left(P_{n}\right)_{n \in \mathbb{N}}$ by Theorem 3.4, the general solution of the difference equation (5.1) has the form $f(n)=f(0) \cdot P_{n}(0)$.

Now we consider the following problem: find all solutions $f: \mathbb{N} \rightarrow \mathbb{C}$ of the difference equation

$$
(n+2) f(n+2)-(2 n+3) f(n+1)+(n+1) f(n)=0
$$

with $f(0)=f(1)$. Observe, that by introducing $g(n)=(n+1) f(n+1)-(n+1) f(n)$ for $n=0,1, \ldots$ we have $g(n+1)-g(n)=0$, which means that $g$ is constant and $g(n)=g(0)=$ $f(1)-f(0)=0$. It follows $(n+1) f(n+1)-(n+1) f(n)=0$, which implies again that $f$ is constant: $f(n)=f(0)$ for each $n$ in $\mathbb{N}$. Then again one can realize that (5.6) is exactly the difference equation

$$
\mathscr{T} f=f
$$

on the Legendre-hypergroup, which is a very special case of (3.2), and can be solved with the method we offered above. Indeed, the characteristic polynomial has the form $Q(\lambda)=\lambda-1$ and the only characteristic root is $\lambda=1$ with multiplicity 1 . According to Theorem 3.4, the general solution of the difference equation (5.6) has the form $f(n)=$ $f(0) \cdot P_{n}(1)$, where $P_{n}$ is the $n$th Legendre-polynomial. As $P_{n}(1)=1$ for each $n$ in $\mathbb{N}$, we have that all solutions of (5.6) satisfying $f(0)=f(1)$ are constant.

We can modify (5.7) to consider

$$
\mathscr{T} f=c \cdot f
$$


where $c$ is a complex parameter. This is the eigenvalue problem for the translation operator $\mathscr{T}$ on any polynomial hypergroup with generating polynomials $\left(P_{n}\right)_{n \in \mathbb{N}}$. In this case the characteristic polynomial is $Q(\lambda)=\lambda-c$ having the only characteristic root $\lambda=c$ with multiplicity 1 . Hence each complex number $c$ is an eigenvalue with the corresponding eigenfunction $n \mapsto P_{n}(c)$.

Example 5.2. The study of higher order difference equations leads naturally to the study of generalized polynomial functions on polynomial hypergroups. We will consider this problem in more details elsewhere, here we work out a simple special case only. Consider the difference equation

$$
\mathscr{T}^{2} f-2 \mathscr{T} f+f=0
$$

or $\Delta^{2} f=0$, where we use the notation $\Delta=\mathscr{T}-I$, and $I$ is the identity operator. The generating polynomials of the underlying polynomial hypergroup are the polynomials $\left(P_{n}\right)_{n \in \mathbb{N}}$. The characteristic polynomial of $(5.9)$ is $Q(\lambda)=\lambda^{2}-2 \lambda+1=(\lambda-1)^{2}$, hence the only characteristic root is $\lambda=1$ with multiplicity 2 . By Theorem 3.4 the general solution of (5.9) has the form

$$
f(n)=A \cdot P_{n}(1)+B \cdot P_{n}^{\prime}(1)=A+B \cdot P_{n}^{\prime}(1)
$$

with arbitrary complex constants $A, B$. We know from the results of [5] that $n \mapsto B$. $P_{n}^{\prime}(1)$ represents a general additive function on the given hypergroup, hence (5.9) can be considered as a characterization of affine functions on polynomial hypergroups - exactly as in the group case.

Example 5.3. Our last example illustrates the application of Theorem 4.1. We consider the difference equation

$$
\mathscr{T}_{2} f-2 \mathscr{T}_{1} f+f=0
$$

on the hypergroup which is generated by the sequences $\left(\alpha_{n}\right)_{n \in \mathbb{N}},\left(\beta_{n}\right)_{n \in \mathbb{N}}$ and $\left(\gamma_{n}\right)_{n \in \mathbb{N}}$. By Theorem 4.1 the general solution of (5.11) can be described with the help of the roots of the polynomial

$$
\lambda \longmapsto P_{2}(\lambda)-2 P_{1}(\lambda)+1,
$$

where $P_{n}$ denotes the $n$th basic polynomial for all $n$ in $\mathbb{N}$. Using the recursive formula for $n=1$ and the property $\alpha_{n}+\beta_{n}+\gamma_{n}=1$ we get

$$
\gamma_{1}\left(P_{2}(\lambda)-2 P_{1}(\lambda)+1\right)=(\lambda-1)\left(\lambda-\left(\gamma_{1}-\alpha_{1}\right)\right)
$$

hence the solutions are the functions

$$
f(n)=A P_{n}(1)+B P_{n}\left(\gamma_{1}-\alpha_{1}\right)=A+B P_{n}\left(\gamma_{1}-\alpha_{1}\right)
$$


with arbitrary complex numbers $A, B$. On the Chebyshev-hypergroup, where the recursive formula for the Chebyshev-polynomials $\left(T_{n}\right)_{n \in \mathbb{N}}$ is

$$
\lambda T_{n}(\lambda)=\frac{1}{2} T_{n+1}(\lambda)+\frac{1}{2} T_{|n-1|}(\lambda)
$$

with $T_{0}(\lambda)=1$ and $T_{1}(\lambda)=\lambda$ the above equation has the form

$$
f(n+4)-2 f(n+3)+2 f(n+2)-2 f(n+1)+f(n)=0
$$

with the initial conditions

$$
f(2)=2 f(1)-f(0), \quad f(3)=f(1) .
$$

The general solution has the form

$$
f(n)=A+B \cdot T_{n}(0)
$$

with arbitrary complex numbers $A, B$, and more explicitly, we can write $f(2 n)=A+$ $B(-1)^{n}$ and $f(2 n+1)=0$ for each $n$ in $\mathbb{N}$. As in this case the problem reduces to a linear homogeneous difference equation with constant coefficients, the same result can be derived from the classical theory. Nevertheless, in the case of the Legendre-hypergroup one obtains a linear homogeneous difference equation with nonconstant coefficients, and the classical methods cannot be directly applied but by the virtue of (5.14) we know that the solutions are the functions

$$
f(n)=A+B \cdot P_{n}\left(\frac{1}{3}\right)
$$

where $P_{n}$ is the $n$th Legendre-polynomial. Similarly, in the case of the Chebyshevhypergroup of the second kind the recursive formula has the form

$$
\lambda U_{n}(\lambda)=\frac{n+2}{2 n+2} U_{n+1}(\lambda)+\frac{n}{2 n+2} U_{|n-1|}(\lambda)
$$

with $U_{0}(\lambda)=1$ and $U_{1}(\lambda)=\lambda$, thus the solutions of (5.11) are the functions

$$
f(n)=A+B \cdot U_{n}\left(\frac{1}{2}\right)
$$

\section{Acknowledgment}

The research was supported by the Hungarian National Foundation for Scientific Research (OTKA), Grant no. T-016846.

\section{References}

[1] K. A. Ross, Hypergroups and signed hypergroups, Harmonic Analysis and Hypergroups (Delhi, 1995) (K. A. Ross, J. M. Anderson, G. L. Litvinov, A. I. Singh, V. S. Sunder, and N. J. Wildberger, eds.), Trends Math., Birkhäuser Boston, Massachusetts, 1998, pp. 77-91. 


\section{Difference equations on discrete polynomial hypergroups}

[2] W. R. Bloom and H. Heyer, Harmonic Analysis of Probability Measures on Hypergroups, de Gruyter Studies in Mathematics, vol. 20, Walter de Gruyter, Berlin, 1995.

[3] L. Gallardo, Some methods to find moment functions on hypergroups, Harmonic Analysis and Hypergroups (Delhi, 1995) (K. A. Ross, J. M. Anderson, G. L. Litvinov, A. I. Singh, V. S. Sunder, and N. J. Wildberger, eds.), Trends Math., Birkhäuser Boston, Massachusetts, 1998, pp. 13-31.

[4] A. L. Schwartz, Three lectures on hypergroups: Delhi, December 1995, Harmonic Analysis and Hypergroups (Delhi, 1995) (K. A. Ross, J. M. Anderson, G. L. Litvinov, A. I. Singh, V. S. Sunder, and N. J. Wildberger, eds.), Trends Math., Birkhäuser Boston, Massachusetts, 1998, pp. 93-129.

[5] Á. Orosz and L. Székelyhidi, Moment functions on polynomial hypergroups, Archiv der Mathematik 85 (2005), no. 2, 141-150.

Ágota Orosz: Institute of Mathematics and Informatics, University of Debrecen, 4010 Debrecen, Hungary

E-mail address: oagota@math.klte.hu 